\title{
Thrombectomy with Conscious Sedation Compared with General Anesthesia: A DEFUSE 3 Analysis
}

(D) C.J. Powers, DD. Dornbos III, (D) M. Mlynash, (DD. Gulati, (DM. Torbey, DS.M. Nimjee, (D) M.G. Lansberg, (D) G.W. Albers, and (iD) M.P. Marks

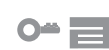

\section{ABSTRACT}

BACKGROUND AND PURPOSE: The optimal patient sedation during mechanical thrombectomy for ischemic stroke in the extended time window is unknown. The purpose of this study was to assess the impact of patient sedation on outcome in patients undergoing thrombectomy 6-16 hours from stroke onset.

MATERIALS AND METHODS: Endovascular Therapy Following Imaging Evaluation for Ischemic Stroke 3 (DEFUSE 3) was a multicenter, randomized, open-label trial of thrombectomy for ICA and M1 occlusions in patients 6-16 hours from stroke onset. Subjects underwent thrombectomy with either general anesthesia or conscious sedation at the discretion of the treating institution.

RESULTS: Of the 92 patients who were randomized to intervention, 26 (28\%) underwent thrombectomy with general anesthesia and 66 (72\%) underwent thrombectomy with conscious sedation. Baseline clinical and imaging characteristics were similar among all groups. Functional independence at 90 days was $23 \%$ for general anesthesia, $53 \%$ for conscious sedation, and $17 \%$ for medical management $(P=$ .009 for general anesthesia versus conscious sedation). Conscious sedation was associated with a shorter time from arrival in the angiosuite to femoral puncture (median, 14 versus 18 minutes; $P=0.05$ ) and a shorter time from femoral puncture to reperfusion (median, 36 versus 48 minutes; $P=.004)$. Sixty-six patients were treated at sites that exclusively used general anesthesia $(n=14)$ or conscious sedation $(n=$ 52). For these patients, functional independence at 90 days was significantly higher in the conscious sedation subgroup (58\%) compared with the general anesthesia subgroup $(21 \%)(P=.03)$.

CONCLUSIONS: Patients who underwent thrombectomy with conscious sedation in the extended time window experienced a higher likelihood of functional independence at 90 days, a lower NIHSS score at 24 hours, and a shorter time from femoral puncture to reperfusion compared with those who had general anesthesia. This effect remained robust in institutions that only treated patients with a single anesthesia technique.

ABBREVIATIONS: $C S=$ conscious sedation; $\mathrm{GA}=$ general anesthesia; $\mathrm{IQR}=$ interquartile range

$\mathrm{T}$ here is controversy regarding optimal anesthesia management during thrombectomy. Currently studied options include general anesthesia (GA), conscious sedation (CS), or local

Received January 29, 2019; accepted after revision April 1.

From the Departments of Neurological Surgery (C.J.P., D.D., S.M.N.) and Neurology (D.G.), Ohio State University Wexner Medical Center, Columbus, Ohio; Departments of Neurology and Neurological Science (M.M., M.G.L., G.W.A.) and Diagnostic Radiology (M.P.M.), Stanford University School of Medicine, Stanford, California; and Department of Neurology (M.T.), University of New Mexico, Albuquerque, New Mexico.

This research was supported financially by the National Institute of Neurologic Disorders and Stroke.

Please address correspondence to Ciarán J. Powers, MD, PhD, N1022 Doan Hall, 410 West 10th Ave, Columbus, $\mathrm{OH} 43220$; e-mail: ciaran.powers@osumc.edu

- Indicates open access to non-subscribers at www.ajnr.org

三 Indicates article with supplemental on-line table.

http://dx.doi.org/10.3174/ajnr.A6059 anesthetic only. In their meta-analysis of 22 studies (3 randomized controlled trials and 19 observational studies) including 4716 patients, Brinjikji et $\mathrm{al}^{1}$ reported higher odds of death and respiratory complications and lower odds of good functional outcomes after thrombectomy for patients who had GA. In contrast, 3 small randomized studies have not shown a difference in outcomes between GA and conscious sedation, though all these studies were performed using thrombectomy within the early time window ( $<6$ hours). ${ }^{2-4}$ The 2018 American Heart Association Guidelines for the early management of ischemic stroke recommend the selection of GA or conscious sedation based on individual patient risk factors, technical aspects of the procedure, and other clinical characteristics. ${ }^{5}$ These guidelines are also based solely on studies of endovascular therapy in the early time window.

The Endovascular Therapy Following Imaging Evaluation for 
Ischemic Stroke 3 (DEFUSE 3) study showed that mechanical thrombectomy for ischemic stroke, between 6 and 16 hours from last known well, plus standard medical therapy resulted in better functional outcome than medical therapy alone for patients with internal carotid artery or proximal middle cerebral artery (M1) occlusion and a target mismatch profile on perfusion imaging. ${ }^{6}$ The use of general anesthesia as opposed to conscious sedation during mechanical thrombectomy was at the discretion of the treating interventionalist.

In this substudy of the DEFUSE 3 trial, we explored the impact of anesthesia management on patient outcome after mechanical thrombectomy in the extended time window.

\section{MATERIALS AND METHODS}

DEFUSE 3 was a multicenter, randomized, open-label, blinded end point trial of thrombectomy for ICA and M1 occlusions in patients who could be treated during an extended time window, 6-16 hours after they were last known well, and who had a favorable ratio between penumbra and core infarct. The local institutional review boards at each site approved the study, and informed consent was obtained from each patient's legal next of kin. Patients were eligible if they had an infarct volume of $<70 \mathrm{~mL}$, a ratio of volume of ischemic tissue to initial infarct volume of $\geq 1.8$, and an absolute volume of potentially reversible ischemia of $\geq 15 \mathrm{~mL}$. Furthermore, patients had an occlusion of the cervical or intracranial internal carotid artery or the proximal middle cerebral artery on noninvasive vascular imaging. Patients with basilar artery occlusions were not included in this study. Ninety-two patients were randomized to the endovascular therapy group, and 90 , to the medical therapy group.

The use of GA as opposed to CS during thrombectomy was at the discretion of the treating providers. The treating center recorded whether GA or CS was used and the reasons for choosing the method of sedation on the primary case report forms. The reasons for choosing GA over CS included routine preference for GA during thrombectomy, interventionalist preference, inability of patients to protect their airways, and excessive patient motion. Assignment of anesthesia choice was based on what was actually performed, not intention-to-treat.

The primary outcome in this analysis of the DEFUSE 3 study was functional independence ( $\mathrm{mRS}$ score of $0-2$ ) at 90 days, comparing GA with CS. Other outcomes included the odds ratio of functional independence at 90 days, median mRS scores at 90 days, NIHSS score at 24 hours and discharge, revascularization efficacy (TICI score), and procedural measures, including median times from symptom onset to imaging, imaging to arrival in the angiosuite, arrival to femoral puncture, and puncture to reperfusion.

\section{Statistical Analysis}

Continuous variables were compared using the Mann-Whitney $U$ test for 2 groups or the Kruskal-Wallis test for 3 groups. We compared frequencies using the $\chi^{2}$ test. Exact statistics were used when expected cell frequencies in a $2 \times 2$ table were $<5$. We used logistic regression or ordinal logistic regression for the multivariable analysis with binary or ordinal outcome measures. Results of the ordinal logistic regression analyses were accepted only if the proportionality test result was not significant. Significance was defined at $\alpha<.05$, and all tests were 2 -sided. The multivariable regression analysis was adjusted for age, NIHSS score, serum glucose level, and time from last known well to randomization. Statistical analysis was performed with the SAS 9.4 (SAS Institute, Cary, North Carolina).

\section{RESULTS}

Ninety-two patients were randomized to intervention, with 26 (28\%) undergoing thrombectomy using GA and 66 (72\%) undergoing thrombectomy with CS. There were only 4 patients with CS before GA ( $15 \%$ of 26 GA cases). Their rate of mRS 0-2 was not different from that in other patients with GA: 1/4 (25\%) versus $5 / 22(23 \%)(P=1.0)$. If we had assigned them to the CS group, then the rates of mRS 0-2 for CS versus pure GA would be $36 / 70$ $(51 \%)$ versus $5 / 22(23 \%)(P=.02)$. This is not meaningfully different compared with the rates of good outcome based on what was actually performed (53\% versus $23 \%$ ).

There were no significant differences among the GA, CS, and medical management (90 patients) groups in age, sex, median NIHSS score, affected hemisphere, or treatment with intravenous tPA (On-line Table). There were also no significant differences among the 3 groups in the imaging characteristics, including volume of ischemic core, volume of hypoperfusion lesion, occlusion site, or ASPECTS score on baseline CT (On-line Table). There were no differences in rates of good collaterals using the Modified Tan scale for the 131 patients who underwent CTA. There were no differences in the first blood pressures on arrival.

Additionally, there were no differences between the GA and CS groups in the history of atrial fibrillation, diabetes mellitus, hypercholesterolemia, myocardial infarction, or prior stroke (On-line Table). There was no difference in the baseline mRS 0-2 among these groups either (On-line Table).

The primary outcome of functional independence, defined as mRS $0-2$ at 90 days, was $23 \%$ for GA, $53 \%$ for CS, and $17 \%$ for medical management (Table). The adjusted common odds ratio of functional independence based on multivariable regression analysis comparing GA versus CS was 0.27 (95\% Confidence Interval $=0.09-0.75)(P=.01)$. The median mRS score at 90 days was 3.5 (interquartile range $[\mathrm{IQR}]=3-5$ ) for thrombectomy with GA, 2 (IQR = 1-4) for thrombectomy with CS, and $4(\mathrm{IQR}=3-6)$ with medical management $(P<.001$ comparing all 3 groups; $P=.05$ for GA versus CS; and $P=.22$ for GA versus medical management) (Table and Figure). Additionally, the NIHSS score at 24 hours (median, 15.5 versus $8 ; P=.03$ ) and at discharge (median, 8 versus $4 ; P=.13$ ) was lower with CS than with GA, though the latter difference was not significant. In addition, the length of stay for patients treated with CS was shorter than for those treated with GA (mean, 5.93 versus 8.02 days; $P=.03$ ), though there were no differences in discharge destination.

Sixty-six patients were enrolled by sites that performed $>1$ thrombectomy and exclusively used GA $(n=14)$ or CS $(n=52)$. While there was no difference in the ordinal mRS scores at 90 days for these patients, functional independence was significantly higher in the CS subgroup (58\%) than in the GA subgroup (21\%) $(P=.033)$. 
Table: Outcome measures for patients in DEFUSE 3

\begin{tabular}{|c|c|c|c|c|c|c|}
\hline Outcome Measures & $\begin{array}{l}\text { Thrombectomy } \\
\text { with GA }(n=26)\end{array}$ & $\begin{array}{l}\text { Thrombectomy } \\
\text { with CS }(n=66)\end{array}$ & $\begin{array}{l}\text { Med Therapy } \\
\quad(n=90)\end{array}$ & $\begin{array}{l}P G A \\
\text { vs CS }\end{array}$ & $\begin{array}{l}P G A \\
\text { vs Med }\end{array}$ & $\begin{array}{l}P C S \\
\text { vs Med }\end{array}$ \\
\hline $\begin{array}{l}\text { Primary outcome: functional independence } \\
\text { at } 90 \text { days (No.) (\%) }\end{array}$ & $6(23)$ & $35(53)$ & $15(17)$ & .01 & .56 & $<.001$ \\
\hline $\begin{array}{l}\text { Median score on modified Rankin Scale at } \\
90 \text { days (IQR) }\end{array}$ & $3.5(3-5)$ & $2(1-4)$ & $4(3-6)$ & .047 & .22 & $<.001$ \\
\hline NIHSS score at $24 \mathrm{hr}$ (IQR) & $15.5(6-20)$ & $8(4-13)$ & $16(11-21)$ & .03 & .31 & $<.001$ \\
\hline NIHSS score at discharge or at days 5-7 (IQR) & $8(4-12)$ & $4(2-11)$ & $15(7-21)$ & .13 & .01 & $<.001$ \\
\hline \multicolumn{7}{|l|}{ Safety outcomes (No.) (\%) } \\
\hline Death at 90 days & $5(19)$ & $8(12)$ & $23(26)$ & .51 & .51 & .04 \\
\hline Symptomatic intracranial hemorrhage & $1(4)$ & $5(8)$ & $4(4)$ & .67 & 1.0 & .5 \\
\hline Early neurologic deterioration & $2(8)$ & $6(9)$ & $12(13)$ & 1.0 & .52 & .41 \\
\hline Parenchymal hematoma type 2 & $3(12)$ & $5(8)$ & $3(3)$ & .68 & .13 & .28 \\
\hline \multicolumn{7}{|l|}{ Imaging outcomes } \\
\hline Median infarct volume at $24 \mathrm{hr}(\mathrm{IQR})(\mathrm{mL})$ & $39.6(21.1-132.3)$ & $33.6(13.8-71.3)$ & $41.0(25.4-106.2)$ & .37 & .83 & .12 \\
\hline Median infarct growth at $24 \mathrm{hr}$ (IQR) (mL) & $26.9(12.9-104.8)$ & $22.3(7.5-55.6)$ & $32.8(18.3-74.8)$ & .35 & .75 & .04 \\
\hline $\begin{array}{l}\text { Reperfusion }>90 \% \text { at } 24 \mathrm{hr} \text { (No.)/(total } \\
\text { No.) (\%) }\end{array}$ & $16 / 21(76)$ & $43 / 54(80)$ & $12 / 68(18)$ & .76 & $<.001$ & $<.001$ \\
\hline $\begin{array}{l}\text { Complete recanalization at } 24 \mathrm{hr} \text { (No.)/ } \\
\text { (total No.) (\%) }\end{array}$ & $17 / 23(74)$ & $48 / 60(80)$ & $14 / 77(18)$ & .56 & $<.001$ & $<.001$ \\
\hline $\begin{array}{l}\text { Reperfusion }>90 \% \text { and/or complete } \\
\text { recanalization at } 24 \mathrm{hr}(\mathrm{No} .) /(\text { total No.) (\%) }\end{array}$ & $17 / 24(71)$ & $48 / 61(79)$ & $14 / 80(18)$ & .44 & $<.001$ & $<.001$ \\
\hline $\mathrm{TICl}$ score of $2 \mathrm{~b}$ or 3 (No.)/(total No.) (\%) & $18 / 26(69)$ & $52 / 66(79)$ & NA & .33 & NA & NA \\
\hline Length of stay at enrolling hospital (days) & $8.02(5.35-12.18)$ & $5.93(3.31-8.85)$ & $8.94(6.17-14.03)$ & .03 & .33 & $<.001$ \\
\hline Discharge destination & & & & .36 & .83 & .48 \\
\hline Home & $3(12)$ & $13(20)$ & $14(16)$ & .39 & .76 & .53 \\
\hline Acute care facility & $2(8)$ & $6(9)$ & $4(4)$ & & & \\
\hline Skilled nursing facility & $7(27)$ & $11(17)$ & $18(20)$ & & & \\
\hline Acute rehab unit & $10(38)$ & $26(39)$ & $35(39)$ & & & \\
\hline Death & $3(12)$ & $2(3)$ & $9(10)$ & & & \\
\hline Other & $1(4)^{\prime}$ & $8(12)$ & 10 (11) & & & \\
\hline
\end{tabular}

Note:-Med indicates medical management; rehab, rehabilitation.

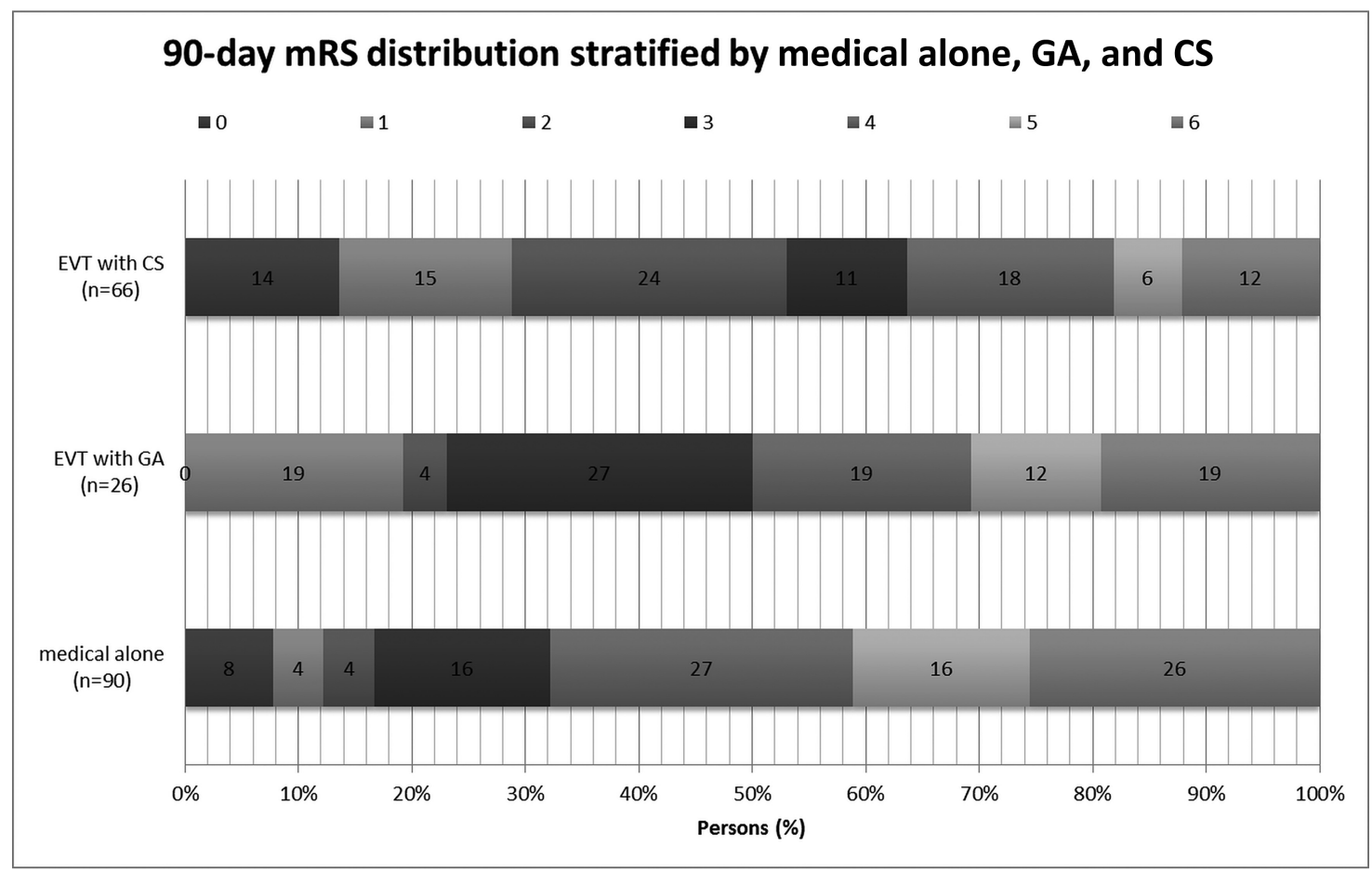

FIGURE. Ninety-day modified Rankin Scale scores for patients in DEFUSE 3 stratified by endovascular therapy (EVT) with conscious sedation, EVT with general anesthesia, and medical therapy alone. 
There were no significant differences in the time from onset of symptoms to qualifying imaging among the 3 groups (On-line Table). For the patients who underwent thrombectomy, there were no significant differences between GA and CS for time to arrival in the angiosuite or from femoral puncture to the first clot removal attempt. However, CS was associated with a shorter time from arrival in the angiosuite to femoral puncture (median, 14 versus 18 minutes; $P=.05$ ) and a shorter time from femoral puncture to reperfusion (TICI $2 \mathrm{~b}$ or 3 as assessed by the core lab) (median, 35 versus 42 minutes; $P=.03$ ).

There were no significant differences in symptomatic intracranial hemorrhage, early neurologic deterioration, or type 2 parenchymal hematoma among the 3 groups (Table). While there was no difference in the death rates at 90 days between GA and CS, there was a significantly lower mortality in CS versus medical management, with an odds ratio of 0.40 (95\% CI, 0.17-0.97; $P=$ $.04)$, but not when comparing GA with medical management $(\mathrm{OR}=0.69 ; 95 \% \mathrm{CI}, 0.23-2.1 ; P=.54)$. There were no differences in infarct volume or infarct growth at 24 hours among the 3 groups. Both GA and CS were associated with higher reperfusion rates compared with medical therapy, without significant differences between the 2 thrombectomy groups.

Regarding relevant serious adverse events, patients undergoing GA had 1 episode of aspiration pneumonia, 1 episode of respiratory arrest, and 1 episode of respiratory failure. Patients undergoing CS had 1 episode of pneumonia and 1 episode of respiratory failure.

\section{DISCUSSION}

In this analysis of the DEFUSE 3 study, the benefit of mechanical thrombectomy performed during the extended time window, between 6 and 16 hours from last known well, for patients with ICA or M1 occlusions and a target mismatch profile on perfusion imaging was apparent in patients who underwent CS but not in those who underwent GA. Specifically, functional independence at 90 days was observed in 53\% of patients treated with CS, but in only $23 \%$ of those treated with GA and $17 \%$ treated by medical management. CS was associated with a slightly shorter time from arrival in the angiosuite to femoral puncture and from femoral puncture to reperfusion. Patients undergoing CS were also noted to have an improved NIHSS score at 24 hours and to some extent on discharge, which could have contributed to the shorter length of stay. Of note, while the median initial NIHSS score was 3 points higher in the GA group, this was not statistically significant.

Because GA versus CS was not randomized, it is possible that patients who underwent thrombectomy with GA were more clinically unstable than those with CS and thus more likely to have a poor outcome. To address this potential bias, we performed an adjusted analysis to account for differences in baseline predictors of clinical outcome. In addition, we noted that many sites typically chose to use either GA or CS as a matter of institutional preference. For sites that routinely used GA, presumably there would be no tendency for less stable patients to receive GA. Therefore, we also analyzed the patients treated at sites that appeared to exclusively use GA or CS. For these 66 patients, the functional outcome was significantly higher in the CS subgroup (58\%) than the GA subgroup (21\%), indicating that this difference is not sin- gularly attributable to a selection bias in which patients with more comorbidities, higher stroke burden, and worsened overall medical status received GA rather than CS.

These results are consistent with post hoc analyses of other thrombectomy trials. Analysis of the Interventional Management of Stroke III (IMS III) trial showed worsened neurologic outcomes and increased mortality after thrombectomy for patients who underwent GA. ${ }^{7}$ Post hoc analysis of the Multicenter Randomized Clinical Trial of Endovascular Treatment for Acute Ischemic Stroke in the Netherlands (MR CLEAN) study showed improved neurologic outcome at 3 months after thrombectomy for patients who did not undergo GA. ${ }^{8}$ Because these were both post hoc analyses, it remains unclear whether these results were due to a patient selection bias.

The DEFUSE 3 results contrast with results from randomized, single-center trials. The Sedation Versus General Anesthesia for Endovascular Therapy in Acute Stroke-Impact on Neurological Outcome (AnStroke) study randomized 90 patients to CS or GA and found no difference in neurologic outcome after 3 months or any differences in blood pressure decline, blood glucose level, $\mathrm{PaCO}_{2}$, time intervals, degree of recanalization, NIHSS score at 24 hours, infarct volume, or hospital mortality. ${ }^{2}$ In The Sedation vs Intubation for Endovascular Stroke TreAtment Trial (SIESTA), 150 patients were randomized to CS or GA, and more patients were functionally independent after 3 months if they had undergone GA, but there was no change in mortality. ${ }^{3}$ The General Or Local Anaestesia in Intra Arterial THerapy (GOLIATH) study randomized 128 patients to CS or GA and showed no difference in infarct growth, with a trend toward better clinical outcome in the GA arm, though this could have been due to the significant difference in successful reperfusion in the GA arm compared with the CS arm (76.9\% versus $60.3 \%, P=.04) .{ }^{4}$ All 3 of these prospective randomized studies were limited by a small number of patients.

There are a number of potential mechanisms through which GA may contribute to worse outcomes following mechanical thrombectomy. GA requires orotracheal intubation, and this additional step may result in a delay in the therapeutic intervention of mechanical thrombectomy. Hypotension and hyperventilation during GA may result in the loss of pial collaterals maintaining the ischemic penumbra. In addition, orotracheal intubation may place the patient at risk for aspiration. Proponents of GA during mechanical thrombectomy cite the decreased patient motion that allows better imaging quality and a lower risk of device-related complications such as vessel perforation, dissection, or subarachnoid hemorrhage. However, it is unclear that better imaging is important for ICA or M1 occlusion, where the anatomy is more consistent compared with other vessels such as the distal middle cerebral artery. Complications such as perforation and subarachnoid hemorrhage have not been significantly different between patients undergoing GA and CS in prior studies ${ }^{2,3}$ or in this study. As a post hoc analysis, the results of this study are limited by the potential for unrecognized selection bias. Specifically, the interventionalist may have chosen GA over CS for patients who seemed more clinically unstable and therefore were more likely to have a worse outcome. We attempted to address this limitation by performing adjusted analyses and analyzing patients who were 
treated at sites that used only GA or CS for their thrombectomy cases, and the observed difference remained robust in this patient cohort as well. There remains a need for further research on the topic of GA versus CS in the extended time window for mechanical thrombectomy. It will be important that future studies control for the duration of the procedure because this may be longer with GA and confound the results.

\section{CONCLUSIONS}

Patients who underwent thrombectomy with conscious sedation in the extended time window experienced a higher likelihood of functional independence at 90 days, a lower NIHSS score at 24 hours, and a shorter time from femoral puncture to reperfusion compared with those who had general anesthesia. This effect remains robust in institutions that only treated patients with a single anesthesia technique.

Disclosures: Michel Torbey—RELATED: Grant: National Institute of Neurological Disorders and Stroke.* Shahid M. Nimjee-UNRELATED: Board Membership: Medtronic, Comments: Acute Ischemic Stroke Medical Advisory Board. Maarten G. Lansberg-RELATED: Grant: National Institute of Neurological Disorders and Stroke.* Gregory W. Albers-UNRELATED: Equity and Consulting: iSchemaView, Consulting: Medtronic; Michael P. Marks—RELATED: Grant: National Institutes of Health-National Institute of Neurological Disorders and Stroke*; UNRELATED: Stock/Stock Options: ThrombxMedical. *Money paid to the institution.

\section{REFERENCES}

1. Brinjikji W, Pasternak J, Murad MH, et al. Anesthesia-related outcomes for endovascular stroke revascularization: a systematic review and meta-analysis. Stroke 2017;48:2784-91 CrossRef Medline
2. Löwhagen Hendén P, Rentzos A, Karlsson JE, et al. General anesthesia versus conscious sedation for endovascular treatment of acute ischemic stroke: the AnStroke Trial (Anesthesia During Stroke). Stroke 2017;48:1601-07 CrossRef Medline

3. Schönenberger S, Uhlmann L, Hacke W, et al. Effect of conscious sedation vs general anesthesia on early neurological improvement among patients with ischemic stroke undergoing endovascular thrombectomy: a randomized clinical trial. JAMA 2016;316:1986-96 CrossRef Medline

4. Simonsen CZ, Yoo AJ, Sorensen LH, et al. Effect of general anesthesia and conscious sedation during endovascular therapy on infarct growth and clinical outcomes in acute ischemic stroke: a randomized clinical trial. JAMA Neurol 2018;75:470-77 CrossRef Medline

5. Powers WJ, Rabinstein AA, Ackerson T, et al; American Heart Association Stroke Council. 2018 Guidelines for the Early Management of Patients with Acute Ischemic Stroke: A Guideline for Healthcare Professionals From the American Heart Association/ American Stroke Association. Stroke 2018;49:e46-110 CrossRef Medline

6. Albers GW, Marks MP, Kemp S, et al; DEFUSE 3 Investigators. Thrombectomy for stroke at $\mathbf{6}$ to $\mathbf{1 6}$ hours with selection by perfusion imaging. $N$ Engl J Med 2018;378:708-18 CrossRef Medline

7. Abou-Chebl A, Yeatts SD, Yan B, et al. Impact of general anesthesia on safety and outcomes in the endovascular arm of Interventional Management of Stroke (IMS) III trial. Stroke 2015;46:2142-48 CrossRef Medline

8. Berkhemer OA, van den Berg LA, Fransen PS, et al; MR CLEAN investigators. The effect of anesthetic management during intra-arterial therapy for acute stroke in MR CLEAN. Neurology 2016;87:656-64 CrossRef Medline 